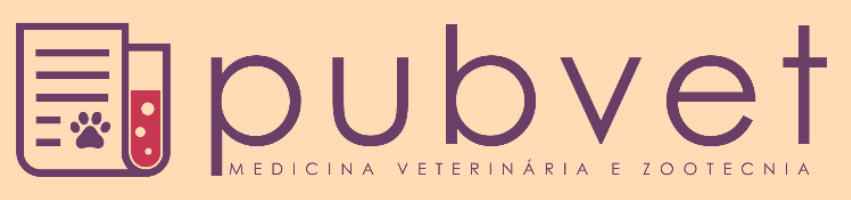

https://doi.org/10.31533/pubvet.v13n9a409.1-7

\title{
Conhecimento sobre radiação ionizante diagnóstica em uma população do sul do Brasil
}

\author{
Carina Burkert da Silva ${ }^{1 *} \bullet$, Thais $\operatorname{Cozza}^{\circ} \operatorname{Sas}^{2} \operatorname{San}^{2} \bullet$, Alana Moraes de Borba ${ }^{2} \bullet$, Bianca \\ Nunes Gomes ${ }^{3} \bullet$, Carolina Litchina Brasil ${ }^{4} \odot$, Daniele Vitor Barboza ${ }^{5}{ }^{\bullet}$, Andreza Bernardi da \\ Silva $^{60}$, Péter de Lima Wachholz ${ }^{7}$, Guilherme Albuquerque de Oliveira Cavalcanti ${ }^{8}$ \\ ${ }^{I}$ Médica Veterinária, Pós-graduada pela Residência em Diagnóstico por Imagem pela Universidade Federal de Pelotas \\ ${ }^{2}$ Médica Veterinária Residente em Diagnóstico por Imagem pela Universidade Federal de Pelotas \\ ${ }^{3}$ Médica Veterinária Pós-graduanda em Diagnóstico por Imagem pelo Instituto Brasileiro de Veterinária \\ ${ }^{4}$ Médica Veterinária Pós-graduada em Diagnóstico por Imagem pelo Instituto Qualittas \\ 5Médica Veterinária Pós-graduada pela Residência em Clínica Médica de Pequenos Animais pela Universidade Federal de Pelotas \\ ${ }^{6}$ Graduanda em Medicina Veterinária pela Universidade Federal de Pelotas \\ ${ }^{7}$ Médico Veterinário Graduado pela Universidade da Região da Campanha \\ ${ }^{8}$ Professor Adjunto do Departamento de Clínicas Veterinárias da Universidade Federal de Pelotas \\ * Autor para correspondência, E-mail: cb.ultrassonografia@gmail.com
}

Resumo. A radiação ionizante é muito utilizada na medicina diagnóstica e aplicada nos exames de radiografia e tomografia computadorizada. Contudo, é capaz de causar efeitos deletérios aos organismos expostos através de mutações genéticas em células somáticas e germinativas, podendo culminar em morte celular, câncer, lesões de pele e glândulas anexas, lesões oftálmicas e malformações e morte fetal. Portanto, através de um questionário com questões objetivas de múltipla escolha disponibilizado na plataforma Formulários Google ${ }^{\circledR}$ avaliou-se o conhecimento da população de Pelotas (RS) sobre os métodos de exames de diagnóstico por imagem, os possíveis efeitos maléficos da exposição à radiação ionizante e levantaram-se dados acerca do uso de proteção radiológica nos serviços de radiologia. Também foram questionados gênero, escolaridade e sobre possuir ou não formação na área da saúde, buscando correlacionar essas informações às respostas. Observou-se que a maior parte dos respondentes não está esclarecida sobre os exames de diagnóstico por imagem e quais utilizam radiação ionizante. A maioria tem conhecimento sobre alguns riscos da radiação ionizante sobre organismo, porém muitos relataram não lhes ser oferecidos equipamentos de proteção radiológica e instrução verbal no momento da realização de exames radiográficos. O presente estudo mostrou a necessidade eminente de se prestar mais esclarecimentos à população sobre o assunto, sobretudo entre profissionais da saúde.

Palavras chave: Diagnóstico por imagem, equipamentos de proteção, radiação ionizante

\section{Knowledge about diagnostic ionizing radiation in a population of southern Brazil}

Abstract. The ionizing radiation it is very used in diagnostic medicine and applied in
radiography and computerizing tomography exams. However, it is capable of cause
deleterious effects in exposed organisms by means of genetic mutation, cancer, skin injuries
and attached glands, ophthalmic injuries and malformation and fetal death. Therefore,
through a quiz of object questions with multiple choices available in Google $\AA$ Forms
platform it was evaluated the knowledge of the population of Pelotas (RS) about methods
of diagnostic imaging exams, the possible harmful effects from ionizing radiation exposure 
and it was collected data about the use of radiological protection in radiological services. Also, it was questioned about genre, scholarity and has or not training in health, looking for correlates this information with the answers. It was noticed that majority of the questioned people are not clear about diagnostic imaging exams and which ones use ionizing radiation. Most of them have knowledge about some risks of ionizing radiation in the body, however many of them reported have not been offered radiological protection equipment and verbal instruction at the moment of perform the radiological exams. The present study shows an imminent necessity of lend more information to the population respect this subject, mostly between health professionals.

Keywords: Imaging diagnostic, protection equipment, ionizing radiation

\section{Conocimiento sobre radiación ionizante diagnóstica en una población del sur de Brasil}

Resumen. La radiación ionizante es muy utilizada en medicina diagnóstica y aplicada en exámenes de radiografía y tomografía computarizada. Entretanto, es capaz de causar efectos dañinos a los organismos expuestos por medio de mutaciones genéticas en células somáticas y germinativas, pudiendo culminar en muerte celular, cáncer, lesiones en la piel y glándulas anexas, lesiones oftálmicas, malformaciones y muerte fetal. Debido a esto, por medio de una encuesta con preguntas objetivas de múltiple opción disponibles en la plataforma Formularios Google ${ }^{\circledR}$ se estudió el conocimiento de la población de la ciudad de Pelotas (RS) sobre los métodos de exámenes de diagnóstico por imagen, los posibles efectos malignos de la exposición a la radiación ionizante y se levantaron datos acerca del uso de protección radiológica en los servicios de radiología. También fueron cuestionados género, escolaridad y sobre tener o no formación en el área de salud, buscando correlacionar estas informaciones a las respuestas. Con este trabajo se observó que gran parte de los encuestados no está esclarecida sobre los exámenes de diagnóstico por imagen y cuales utilizan radiación ionizante. La mayoría tiene conocimiento de algunos riesgos de la radiación ionizante sobre el organismo, sin embargo, muchos relataron no haberles ofrecido equipamiento de protección radiológica, tampoco instrucción verbal al momento de realizar los exámenes radiológicos. El presente estudio demostró la necesidad inminente de brindar más esclarecimientos a la población acerca del tema, principalmente entre profesionales de la salud.

Palabras clave: Diagnóstico por imagen, equipamientos de protección, radiación ionizante

\section{Introdução}

Radiação é a propagação de energia em forma de ondas eletromagnéticas, às quais correspondem a variações no espaço e tempo no campo eletromagnético (Catalão, 2010). A radiação pode ser dividida em dois tipos: ionizante e não ionizante. A primeira é um tipo de radiação que possui energia suficiente para ionizar os átomos e moléculas de um organismo vivo com as quais interagem; já a radiação não ionizante não possui energia para ionizar átomos e moléculas. Embora as radiações não ionizantes interajam de uma maneira diferente que as radiações ionizantes, isto não as torna inócuas (Factor, 2005). A radiação ionizante é amplamente utilizada na medicina para fins terapêuticos e diagnósticos, sendo a radiografia e a tomografia computadorizada os principais exemplos de métodos de diagnóstico por imagem que a utilizam.

A interação da radiação ionizante com o organismo humano é chamada "efeito biológico", que pode se manifestar de duas maneiras. Uma delas é o efeito determinístico, causado pela alta dose de radiação, levando a célula à perda parcial ou total de sua função biológica, ou seja, morte celular. A outra forma é o efeito estocástico, onde a exposição à pequenas doses de radiação ao longo do tempo podem causar mutações genéticas. Se a mutação for células germinativas, ocorre mudança hereditária; se a mutação for às células somáticas, há probabilidade do desenvolvimento de câncer, principalmente em tecido mamário, gônadas, medula óssea e tecido linfático. Casos de aborto são relatados, principalmente nas primeiras semanas do desenvolvimento embrionário, porém malformações fetais são os principais riscos 
da exposição durante a gestação. Perda de pelos e alterações de visão também são efeitos em curto prazo causados pela radiação, portanto para minimizar o efeito da radiação são de suma importância o conhecimento e a utilização dos equipamentos de radioproteção (Dimenstein \& Hornos, 2001; IAEA, 2014).

A radioproteção visa à obtenção de um exame de excelente qualidade diagnóstica com o mínimo de exposição do paciente, do tutor do animal e dos funcionários envolvidos (Thrall, 2013). A medicina veterinária não difere da medicina humana na questão das normativas de radioproteção, como a utilização de equipamentos de proteção individual (EPI) plumbíferos (luvas, avental, óculos, protetor de tireoide), dosimetria e baritagem de sala (Kolber, 2006). O uso de vestimentas de proteção radiológica implica na redução de dose absorvida durante o exame radiográfico, sendo as regiões que apresentaram maior redução da dose são as gônadas e a tireoide (Hohl et al., 2005).

Objetivou-se com esta pesquisa avaliar o conhecimento sobre os exames de diagnóstico por imagem e seus possíveis riscos à saúde na população de Pelotas - RS.

\section{Material e métodos}

Foram realizadas entrevistas na população do município de Pelotas, utilizando um questionário estruturado com 11 questões relacionadas ao grau de escolaridade, gênero e exames de diagnóstico por imagem. O questionário foi aplicado pelo sistema de Formulários Google ${ }^{\circledR}$, disponível online e divulgado em redes sociais. As questões apresentavam caráter objetivo de múltipla escolha, tendo o convidado liberdade total para negar sua participação. A pesquisa visou levantar dados sobre o conhecimento da população sobre diagnóstico por imagem, e sobre a obediência às normas de radioproteção pelos serviços de radiodiagnóstico, tanto na medicina humana quanto na medicina veterinária. As questões abordadas incluíram perguntas sobre a realização dos exames de imagem, o uso de proteção plumbífera durante exames radiológicos, exames que utilizam radiação ionizante e riscos que a radiação ionizante apresenta à saúde. Foram indagados gênero, nível de escolaridade dos participantes e questionou-se sobre possuir formação em área da saúde ou não, buscando correlacionar estas informações ao grau de conhecimento dos respondentes acerca do tema. O tamanho da amostra foi definido a partir da população de Pelotas de aproximadamente 330 mil pessoas no último censo do ano de 2010 , objetivando nível de confiança de $95,5 \%$ e margem de erro de 5\%, com porcentagem de 50\% para ocorrência do fenômeno. O cálculo amostral para população finita teve o resultado de 384 respondentes, e o presente estudo obteve um total de 395 respostas.

Os dados obtidos foram submetidos à análise descritiva e os efeitos das variáveis independentes (sendo elas: gênero, escolaridade e formação na área da saúde) sobre as variáveis dependentes (sendo elas: resposta correta para exames que utilizam radiação ionizante, marcar acima de 4 opções corretas ou acima de 3 opções incorretas de riscos da radiação ionizante) foram avaliados pelo teste QuiQuadrado com intervalo de confiança em $95 \%$, considerando-se associação positiva com valor de $\mathrm{P} \geq$ 0,05. As analises estatísticas foram feitas pela da plataforma OpenEpi (Dean et al., 2014).

\section{Resultados e discussão}

A maioria dos participantes foi do gênero feminino, perfazendo 75,9\% das respostas. Do total de respondentes, $18,2 \%$ tinha grau de escolaridade até ensino médio completo e $81,7 \%$ possuiam acima de ensino superior. Dos 395 respondentes, 31,8\% possuem formação em área da saúde.

Quando questionados sobre quais exames acreditam utilizar radiação ionizante, a maioria dos respondentes $(89,1 \%)$ relatou radiografia, seguido de ressonância magnética $(60,5 \%)$ e tomografia computadorizada $(49,3 \%)$. Dezessete vírgula quatro por cento dos indivíduos responderam que exames de ultrassonografia utilizam radiação ionizante, $6,1 \%$ responderam eletrocardiografia, 1,5\% endoscopia e $2 \%$ dos indivíduos acreditam que nenhum exame faz uso de radiação ionizante.

A questão sobre quais exames utilizam radiação ionizante obteve $9,1 \%$ de respostas corretas sobre o total de participantes. Dentre o total de respondentes do gênero masculino, 17,9\% marcaram as opções corretas de exames que utilizam radiação ionizante, obtendo maior número de acerto em relação às mulheres, que obtiveram $6,3 \%$ de acertos, implicando em uma diferença altamente significativa no percentual de respostas corretas $(\mathrm{P}=0,0006)$. As respostas corretas dentre o total de indivíduos que 
possuíam até ensino médio completo foi $8,3 \%$, enquanto que dos que possuíam a partir de ensino superior, as respostas corretas totalizaram 9,29\% $(\mathrm{P}=0,799)$. Dos profissionais da área da saúde, 11,9\% deles acertaram quais exames utilizam radiação ionizante, contra 7,8\% de acertos entre profissionais de outras áreas $(\mathrm{P}=0,187)$. $\mathrm{O}$ nível de escolaridade dos respondentes não influenciou nos acertos desta questão, tampouco a formação em área da saúde, não implicando em uma diferença estatística para o percentual de respostas corretas para esta questão. Além de não obterem maior percentual de respostas corretas em relação aos demais participantes, $61,4 \%$ do total de respondentes com formação em área da saúde marcaram a opção ressonância magnética como exame que utiliza radiação ionizante.

A radiação é capaz de induzir mutações genéticas em tecidos como gônadas, mamas e tireoide, dando origem a neoplasias. A morte do embrião pode ocorrer se houver exposição nas primeiras duas semanas após a concepção. Posterior a esse período, o feto torna-se menos sensível, onde um exame radiográfico simples geralmente não produz radiação ionizante suficiente para causar sua morte. Todavia, se uma gestante for submetida a exames que gerem maiores doses de radiação ionizante, como múltiplas radiografias ou tomografia computadorizada, por exemplo, o risco de aborto existe (Paula \& Medeiros, 2001; Videira \& Nogueira, 2002). Sendo assim, prevalecem os riscos de malformações fetais causados pela radiação ionizante principalmente no primeiro trimestre de gestação (Dimenstein \& Hornos, 2001). As diretrizes da Agência Internacional de Energia Atômica (IAEA, 2014) apontam que pele e olhos são órgãos especialmente sensíveis aos efeitos da radiação devido à exposição direta por serem estruturas superficiais e pouco protegidas. $\mathrm{O}$ cristalino não possui um sistema de remoção de células lesadas, o que pode progressivamente levar ao aparecimento de catarata em uma exposição crônica. Os efeitos da radiação sobre a pele e anexos incluem inflamação, descamação e destruição de glândulas sebáceas e folículos pilosos. O Departamento de Saúde e Serviços Humanos dos Estados Unidos (1991) atenta para o fato de que pequenas doses de radiação são suficientes para causar efeitos deletérios no organismo exposto, porém não foram encontrados dados sobre casos recentes de óbitos de profissionais da área e de clientes submetidos a estas.

As alternativas possíveis de serem assinaladas sobre os riscos do uso indiscriminado de radiação ionizante sobre o organismo estão ilustradas na Figura 1.

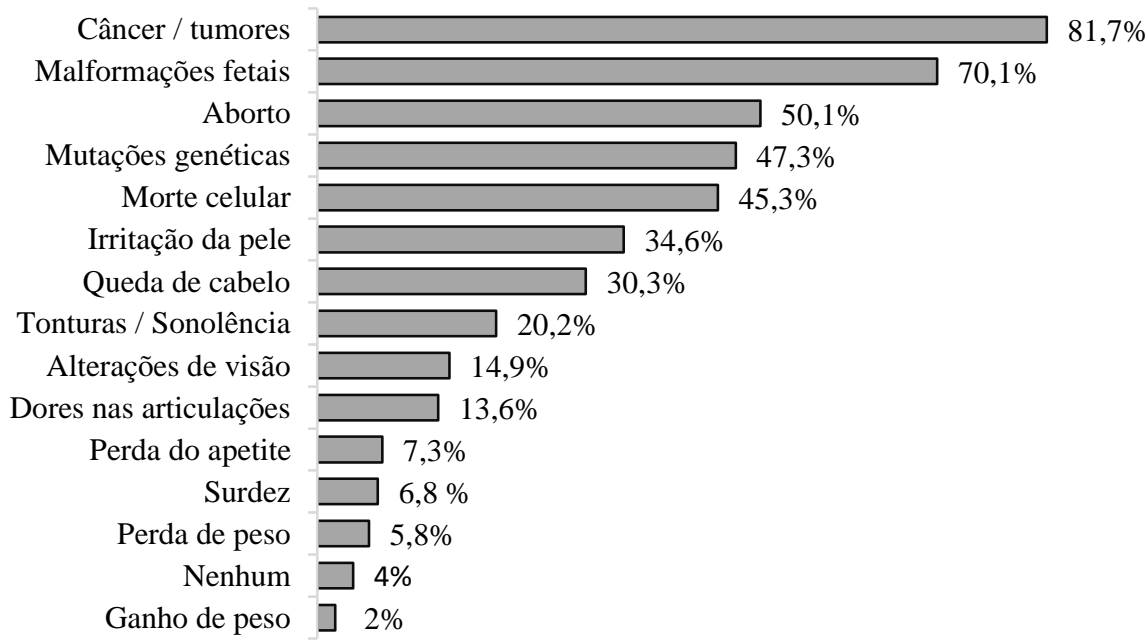

Figura 1. Resultado obtido por meio de questionário de múltipla escolha sobre os possíveis riscos e efeitos da radiação ionizante sobre organismos vivos, onde apenas as respostas câncer/tumores, malformações fetais, aborto, mutações genéticas, morte celular, irritação da pele, queda de cabelo, e alterações de visão foram consideradas respostas corretas.

As duas opções selecionadas pela grande maioria dos respondentes como complicações da radiação ionizante foram "câncer/tumores", obtendo 81,7\% das respostas e "malformações fetais" com 70,1\%. Seguidas das opções "aborto", selecionada por 50,1\% dos respondentes, "mutações genéticas" com $47,3 \%$ e "morte celular" com $45,3 \%$ das respostas.

Considerando haver oito respostas corretas (sendo elas: câncer/tumores, malformações fetais, aborto, mutações genéticas, morte celular, irritação da pele, queda de cabelo, e alterações de visão) e 6 respostas 
erradas (sendo elas: tonturas/sonolência, dores nas articulações, perda do apetite, surdez, perda de peso e ganho de peso), as respostas foram classificadas em um sistema de número de acertos e erros, onde cada opção marcada vale 1 ponto. Os números de acertos e erros de estão ilustrados na Tabela 1.

Tabela 1. Números de acertos e erros dos riscos e efeitos da radiação ionizante sobre organismos vivos

\begin{tabular}{llcc}
\hline Acertos & $\mathrm{N}$ & Erros & $\mathrm{N}$ \\
\hline 1 & 54 & 1 & 56 \\
2 & 61 & 2 & 27 \\
3 & 50 & 3 & 14 \\
4 & 64 & 4 & 5 \\
5 & 57 & 5 & 3 \\
6 & 46 & 6 & 5 \\
7 & 36 & & \\
8 & 11 & & \\
\hline
\end{tabular}

Do total de respondentes do estudo, $54,1 \%$ obtiveram 4 ou mais acertos sobre riscos da radiação ionizante. O percentual do total de homens que marcou acima de quatro alternativas corretas superou as mulheres. Do total de mulheres, 50\% acertaram acima de 4 opções; dentre o total de homens, $67.0 \%$ (P $=0,0030$ ). Do total de indivíduos com grau de escolaridade acima de ensino superior, $58,5 \%$ deles acertaram mais de 4 alternativas, enquanto o total dos que possuíam até ensino médio completo, apenas $34,7 \%$ acertaram tiveram mais acertos $(\mathrm{P}=0,0002)$. Houve mais indivíduos que marcaram mais de 4 opções corretas dentre os profissionais da saúde, perfazendo $72,2 \%$ do total destes, contra $45,7 \%$ dentre o total dos demais respondentes $(\mathrm{P}=0,0000008)$. Portanto, as variáveis como maior grau de escolaridade e formação em área da saúde interferiu positivamente na porcentagem de acertos desta questão, apresentando diferença altamente significativa.

O total de respondentes que pontuaram três ou mais erros sobre riscos da radiação ionizante foi $6,8 \%$. Do total de homens, $15,7 \%$ deles obteve este índice de 3 ou mais erros; do total das mulheres, $4 \%$ (P = 0,00007). Dos indivíduos com até ensino médio completo, 2,7\% marcaram até 3 alternativas erradas, enquanto que dos que possuem graduação, $7,7 \%(\mathrm{P}=0,131)$. Dentre o total de profissionais da saúde, $7,1 \%$ deles marcaram 3 ou mais alternativas erradas, enquanto profissionais de outras áreas, $6,6 \%(\mathrm{P}=$ $0,868)$. Estatisticamente, grau de escolaridade ou formação em área da saúde não apresentou influência sobre o índice de erros. Todavia, novamente os homens superaram as mulheres em seu percentual de pontuação.

Os indivíduos do gênero masculino obtiveram maiores índices de acerto em relação às mulheres. $\mathrm{O}$ grau de escolaridade entre mulheres e homens deste estudo não apresentou diferença estatística, onde $83,3 \%$ das mulheres possuem ensino superior, e os homens, 76,8\% $(\mathrm{P}=0,153)$. A formação em área da saúde tampouco variou sua distribuição entre os indivíduos, já que 33,6\% dos homens e $31,33 \%$ das mulheres alegaram ser da área da saúde $(\mathrm{P}=0,668)$. Sendo assim, o estudo não conseguiu justificar o maior percentual de acertos entre os respondentes masculinos. Não se conseguiu justificar porque o desempenho dos homens foi superior às mulheres, não havendo correlação do gênero masculino com as outras variáveis. Todavia, os homens também marcaram mais opções erradas em relação aos riscos da radiação ionizante.

A maior parte dos indivíduos da área da saúde está satisfatoriamente informada a respeito dos riscos da exposição à radiação ionizante. Porém, o fato destes profissionais da saúde não estarem devidamente esclarecidos sobre quais exames utilizam radiação ionizante é especialmente preocupante ao pensar na possibilidade destes exames serem erroneamente explicados à população, e as normas de radioproteção ser negligenciadas. Estes dados estão de acordo com os observados por Huhn \& Vargas (2016) que constataram que as equipes multiprofissionais de saúde que exercem suas atividades nos setores hospitalares de diagnóstico radiológico frequentemente não têm conhecimento dos conceitos de saúde, risco operacional e segurança. Spyrides (2000) também percebeu negligência na proteção radiológica dos pacientes por parte de profissionais, além de desconhecerem a legislação que regulamenta a proteção radiológica. 
Sobre uso de proteção radiológica durante a realização de exames de radiografia, acima da metade dos indivíduos (57.0\%) que se submeteram a estes exames relatam não lhe terem sido oferecidos algum equipamento de proteção radiológica. Já quando questionados sobre a proteção radiológica ao acompanhar o exame de seu animal de estimação, 68\% dos tutores que já acompanharam exames relatam que lhe foi oferecido o uso de proteção. Sabendo-se dos efeitos que radiação ionizante é capaz de causar ao organismo, a proteção radiológica consiste em medidas essenciais a fim de proteger os pacientes e demais pessoas contra exposição significativa e contínua à radiação resguardando-os dos efeitos biológicos deletérios (Sherer et al., 2017).

Apenas $25 \%$ dos indivíduos que se submeteram à exames de radiografia receberam alguma explicação sobre a importância de utilizar a proteção radiológica durante a realização destes exames. Já na medicina veterinária, $42 \%$ dos acompanhantes de animais relataram ter recebido algum tipo de explicação, resultado que demonstra que os profissionais da radiologia veterinária estão mais preocupados e conscientes da sua responsabilidade sobre o usuário dos exames de imagem, porém não em números desejáveis. Quanto à observação da presença de cartazes explicativos nas salas de exame, em medicina humana, $46.0 \%$ dos usuários alegaram ter observado cartazes, e em medicina veterinária, $49.0 \%$, não se observando diferença entre os dois grupos. Um estudo em medicina humana demonstrou que os pacientes usuários de exames de radiografia desconhecem as normas e meios de proteção, assim como não têm interesse em ler as informações sobre o uso de equipamentos de proteção individual e o risco da exposição (Nobre Neto \& Sousa, 2011). Essas informações reforçam a importância de fornecer orientação verbal aos usuários de exames radiológicos, uma vez que menos da metade relatou observar os cartazes dispostos dentro e fora das salas de exames radiológicos.

\section{Conclusão}

Neste estudo, boa parte dos participantes demonstrou conhecer alguns riscos da exposição à radiação ionizante, sobretudo indivíduos mais escolarizados e profissionais da área da saúde. Entretanto, a maioria dos respondentes não sobre quais exames de imagem utilizam radiação ionizante. Em geral, os homens obtiveram maior percentual de respostas corretas. Muitos respondentes relataram não ter utilizado equipamentos de proteção radiológica nem recebido orientação verbal no momento da realização de exames radiográficos.

O presente estudo mostrou a necessidade de se fomentar mais medidas esclarecedoras acerca dos tipos de radiação utilizada pelos exames de diagnóstico por imagem, bem como os métodos de proteção radiológica para a população em geral. Ficou clara a importância da educação continuada entre os profissionais da área da saúde, em especial aos que atuam em serviços de diagnóstico por imagem, e a necessidade de reforçar conceitos básicos de radiologia e normas de proteção radiológica.

\section{Referências bibliográficas}

Catalão, J. P. S. (2010). Campos eletromagnéticos em sistemas biológicos apontamentos das aulas teóricas: Universidade da Beira Interior.

Dean, A. G., Sullivan, K. M. \& Soe, M. M. (2014). OpenEpi: open source epidemiologic statistics for public health, version (Vol. 3).

Dimenstein, R. \& Hornos, Y. M. M. (2001). Manual de proteção radiológica aplicada ao radiodiagnóstico. Editora Senac.

Factor Segurança LDA. (2005). Radiações Ionizantes e não ionizantes. Kéramica. 270:1-2. jan./fev. Disponível em: https://docplayer.com.br/1487104-Publicacoes-tecnometal-n-o-150-janeirofevereiro-de-2004-keramica-n-o-270-janeiro-fevereiro-de-2005.html. Acesso em: 23/01/2019.

Hohl, C., Mahnken, A. H., Klotz, E., Das, M., Stargardt, A., Mühlenbruch, G., . . Wildberger, J. E. (2005). Radiation dose reduction to the male gonads during MDCT: the effectiveness of a lead shield. American Journal of Roentgenology, 184(1):128-130.

Huhn, A. \& Vargas, M. A. O. (2016). Plano de proteção radiológica e responsabilidade ética. Brazilian Journal of Radiation Sciences, 4(1A):2-7.

IAEA. (2014). International Atomic Energy Agency. International Atomic Energy Agency Safety Standards. Radiation Protection and Safety of Radiation Sources: International Basic Safety Standards - General Safety Requirements. Vienna: Interim. 
Kolber, M. R. e. M. V. I. N., A.I. ( $1^{\text {a }}$ ed.) Tecnologia Radiológica e Diagnóstico por Imagem. São Paulo: Difusão, 4:123-156. (2006). Radiologia em Medicina Veterinária. In A. I. Nobrega (Ed.), Tecnologia Radiológica e Diagnóstico por Imagem (pp. 123-156). São Paulo, Brasil: Difusão.

Nobre Neto, P. G. \& Sousa, G. M. S. F. (2011). Comunicação em saúde: conhecimento de risco relacionado ao procedimento de diagnóstico por imagem. Revista de Pesquisa em Saúde, 12(3):2227.

Paula, L. C. \& Medeiros, R. B. (2001). Exposiçäo à radiaçäo no período pré-natal. Folha Médica, 120(4):213-219.

Sherer, M. A. S., Visconti, P. J., Ritenour, E. R. \& Haynes, K. (2017). Radiation protection in medical radiography. St. Louis, USA: Elsevier.

Spyrides, K. S. (2000). Avaliação do controle de qualidade e proteção radiológica na cidade do Rio de Janeiro. PhD, Universidade Estadual de Campinas, Campinas, São Paulo, Brasil.

Thrall, D. E. (2013). Textbook of veterinary diagnostic radiology. Philadelphia: Elsevier Health Sciences.

Videira, A. I. \& Nogueira, A. (2002). Exposição às radiações ionizantes na gravidez: Efeitos biológicos no embrião e no feto. Arquivos da Maternidade Dr. Alfredo da Costa, 18(2):66-71.

Recebido: 17 de julho, 2019.

Aprovado: 23 de agosto, 2019.

Publicado: 17 de outubro, 2019.

Licenciamento: Este artigo é publicado na modalidade Acesso Aberto sob a licença Creative Commons Atribuição 4.0 (CC-BY 4.0), a qual permite uso irrestrito, distribuição, reprodução em qualquer meio, desde que o autor e a fonte sejam devidamente creditados. 